\title{
Experimental Study of Governing Parameters in Pitching and Plunging Airfoil at Low Reynolds Number
}

\author{
Yeon Sik Baik ${ }^{1}$, Jonathan M. Rausch ${ }^{2}$, Luis P. Bernal ${ }^{3}$, and Wei Shyy ${ }^{4}$ \\ University of Michigan, Ann Arbor, MI \\ Michael V. Ol ${ }^{5}$ \\ Air Force Research Lab, Wright-Patterson AFB
}

\begin{abstract}
An experimental study of pitching and plunging airfoil at $R e=10,000$ and pure sinusoidal effective angle of attack motion is presented in this paper. The experiments were conducted at the University of Michigan low-turbulence water channel facility using 2D phase-averaged particle image velocimetry (PIV). The effect of non-dimensional parameters governing pitching and plunging motion such as Strouhal number $(S t)$, reduced frequency $(k)$, and the plunge amplitude $\left(h_{0}\right)$ is investigated for the same effective angle of attack kinematics. The formation of a leading edge vortex (LEV) and a trailing edge vortex (TEV) is observed for all the cases studied. The formation phase of the LEV was found to be dependent on $k$; the LEV formation is delayed for higher $k$ value. It is found that for cases with the same $k$ the velocity profiles normal to the airfoil surface closely follow each other in all cases independent of pitch rate and pivot point effect. Analysis on the locations of the LEV core based on the Q-criterion and local streamline patterns helps identify the trajectory of the LEV core with respect to the airfoil. Additionally, a trend in the LEV circulation was observed.
\end{abstract}

\section{Nomenclature}

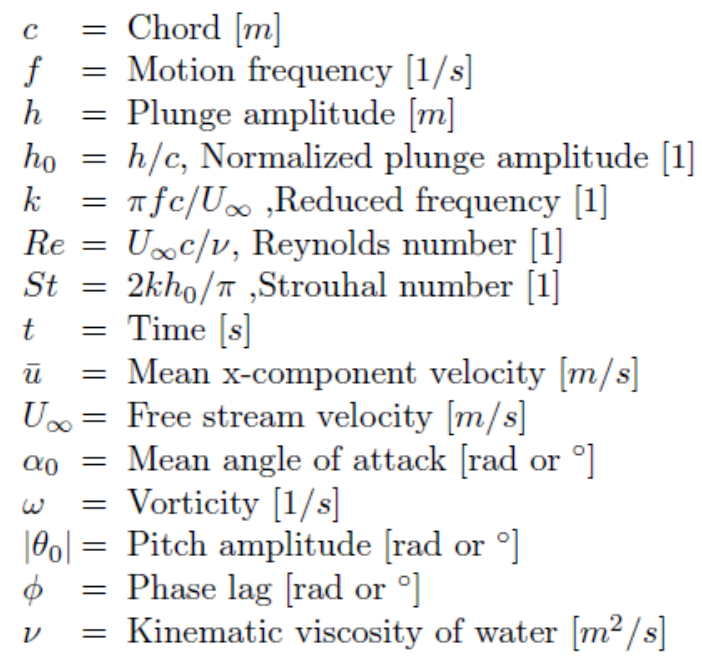

\footnotetext{
1. Graduate Research Assistant, University of Michigan, Department of Aerospace Engineering, yeonb@umich.edu

2. Graduate Research Assistant, University of Michigan, Department of Aerospace Engineering, rauschjm@umich.edu

3. Associate Professor, University of Michigan, Department of Aerospace Engineering, lpb@umich.edu

4. Clarence "Kelly" Johnson Collegiate Professor and Chair, University of Michigan, Department of Aerospace Engineering, weishyy@umich.edu

5. Aerospace Engineer, Air Vehicles Directorate, Wright-Patterson AFB, Michael.Ol@wpafb.af.mil
} 


\section{Introduction}

The unsteady aerodynamics of pitching and plunging wings has been of interest to biologists and aerodynamicists for possible application to Micro Air Vehicle (MAV) design. MAVs are characterized by low $R e$ and the recent monograph by Shyy et al. ${ }^{1}$ provides a detailed review of the subject. The study of periodic pitching and plunging wings which is more relevant to steady flight of birds has been investigated using flow visualization, laser diagnostics, and force measurements ${ }^{2}$. Periodic formation and shedding of large scale leading edge vortices (LEV), separation and reattachment, laminar-to-turbulent transition, and near-wall pressure and velocity variations are several of the phenomena encountered in periodic pitching and plunging wing aerodynamics ${ }^{3-5}$.

Also of interest is the phenomenon of dynamic stall in helicopter blade aerodynamics ${ }^{6}$. The formation of a leading edge vortex during dynamic stall results in an increase in lift coefficient well beyond the static stall angle of attack. Although the reported dynamic stall occurs at Re much larger than relevant to MAV applications, it provides useful insights for low Re. The formation of leading edge vortices are prominent features in the aerodynamics of biological fliers and are believed to be critical to

force generation $^{7-9}$. Flow visualization experiments and detailed flow field measurements of pure pitching, pure plunging, and combined pitching and plunging airfoils have been reported by several researches at the $R e$ regime ranging between $\mathrm{O}\left(10^{3}\right)$ to $\mathrm{O}\left(10^{4}\right)^{10-14}$. Different airfoil kinematics were used, but the basic observation is that the Strouhal number $(S t)$, which is a combination of frequency and amplitude of the motion, determines the drag-producing, neutral, or thrust-producing wake profiles. Anderson et al. ${ }^{15}$ reported that the range of $S t$ for optimal thrust production is 0.25 and 0.40 for harmonically pitching and plunging airfoils using particle image velocimetry (PIV) and direct force measurement. This range of $S t$ is further supported by the data on birds, bats and insects at cruise conditions ${ }^{16}$.

In the present work we extend recent studies ${ }^{17-19}$ of the aerodynamics of pitching and plunging airfoils to consider the effect of frequency and amplitude of motion for fixed airfoil motion kinematics. In previous work we considered two motion kinematics: shallow stall and deep stall cases, and focused on $R e$ number efffects. In the present work we consider the deep stall case and a fixed $R e$ of 10,000. The time history of effective angle of attack, which is the angle of attack in a frame of reference moving with the pivot point of the airfoil, is fixed and the frequency and amplitude of the motion are varied. We explore the resulting two-dimensional space of reduced frequency and motion amplitude ratio, and resulting changes in St. As noted by Anderson et al. ${ }^{15}$ as $S t$ is increased a pure sinusoidal plunge motion does not result in a pure sinusoidal effective angle of attack. In the present case the effective angle of attack time history was forced to be a pure sinusoid by changes in the plunge motion and therefore the motion kinematics were the same for all cases considered and only the reduced frequency and motion amplitude were varied. A rigid flat plate airfoil as a representative airfoil shape was considered which eliminated the effect of camber and wing flexibility, and it will be considered in other studies.

\section{Experimental Setup}

Experiments were conducted in the low-turbulence water channel at the University of Michigan. The low-turbulence water channel has a test cross-section of $61 \mathrm{~cm}$ wide by $61 \mathrm{~cm}$ high, and free stream velocity ranging from $5 \mathrm{~cm} / \mathrm{s}$ to $40 \mathrm{~cm} / \mathrm{s}$ with a turbulence intensity of approximately $1 \%$. The turbulence intensity was measured by taking particle image velocimetry (PIV) images in the free stream for a period comparable to the duration of the unsteady tests while maintaining the water channel operating at a 
constant motor speed. The short time turbulence intensity computed by averaging over a time period on the order of the test section residence time is less than $0.5 \%$, significantly lower than the long time average. The larger value of the long time average is associated with very low frequency sloshing in the facility.

The airfoil section used in this study is a flat plate with chord of $152 \mathrm{~mm}$. It has $2.3 \%$ thickness and has rounded leading and trailing edges with radius equal to half-thickness. The flat plate model was fabricated from stainless steel with polished surface to minimize the diffuse reflection from the laser beam, hence reducing the glare near the surface. The model spanned the depth of the water channel test section and the distance between the model and the bottom of the test section was approximately $1 \mathrm{~mm}$. The vertically mounted models have an attached end plate that is used to minimize free surface effects. This cantilevered mounting scheme results in some model tip deflection due to aerodynamic loading since it is only supported at one end. However, at $\operatorname{Re}$ of 10,000 , the tip deflection is negligible. Figure 1 illustrates the mounting scheme and motor system used at the University of Michigan water channel.

Top View

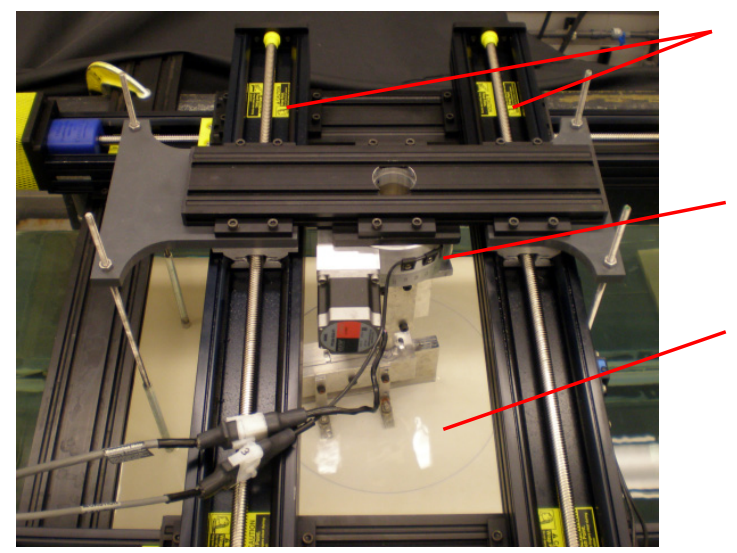

Side View

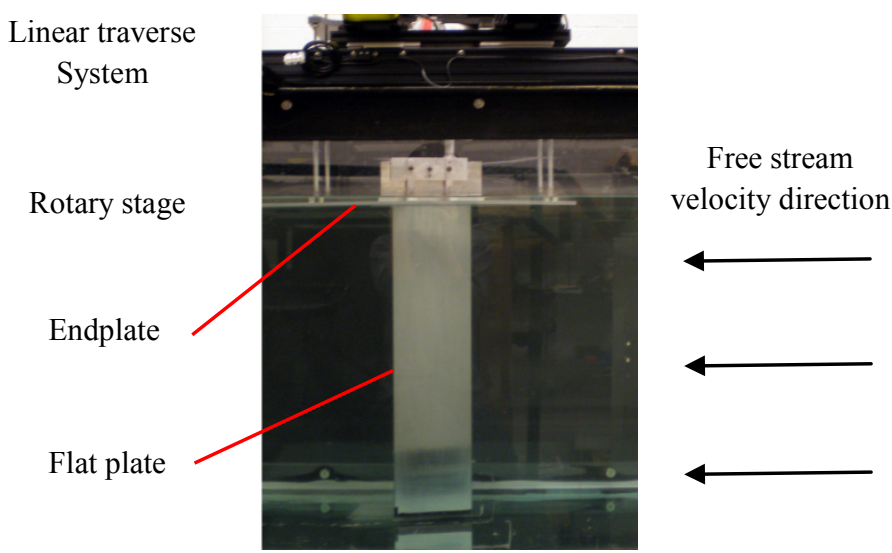

Figure 1. Water channel facility at the University of Michigan.

For qualitative data analysis dye flow visualization was used. The dye flow visualization system consists of seven uniformly distributed dye streams introduced a half chord upstream of the leading edge of the model. Two syringe pumps are used to introduce the dye stream at the same speed as the water channel flow speed in order to minimize disturbances produced by the wake of the dye injection system. The dye streams are held fixed and the airfoil is pitched and plunged within the width of the dye streams. The resulting streaklines capture flow direction and recirculation zones produced by the pitching and plunging motion of the airfoil.

The PIV technique is used for quantitative flow analysis. The PIV system includes a doublepulsed Nd-YAG laser (Spectra Physics PIV 300), light sheet formation optics, two dual frame digital cameras (Cooke Corp. PCO.4000), computer image acquisition system and control electronics. The airfoil motion is produced by a rotary stage (Velmex B4872TS Rotary Table) for the pitch motion, a linear traverse (Velmex 20-inch BiSlide) for the plunge motion, a linear traverse (Velmex 40-inch BiSlide) for the axial motion, and the associated computer control system (Velmex VXM-1-1 motor control). For PIV image acquisition, the water channel was seeded with $3 \mu \mathrm{m}$ diameter Titanium Dioxide particles (SigmaAldrich). A small amount (8 drops) of a dispersant (DARVAN C-N, Vanderbilt) was used to produce a 
uniform distribution of particles and to help maintain the particles in suspension for long periods of time, typically on the order of several days. The cameras were installed underneath the water channel test section and were equipped with Nikon 105-mm Micro-Nikkor lenses to produce a magnification of approximately 16 pixels $/ \mathrm{mm}$. With this magnification the time between exposures was adjusted to produce a nominal particle displacement of 6 pixels at the free stream velocity for all cases. The PCO.4000 camera frame size is 4008 by 2672 pixels, which for the present magnification corresponds to a physical image size of 250 by $167 \mathrm{~mm}$ in the flow. The accuracy of the axial Velmex BiSlide traverse is $0.00635 \mathrm{~mm}$, which corresponds to approximately 0.1 pixel at the present magnification.

In the present measurements the velocity field at specific phases of the airfoil motion were recorded and used to calculate phase-averaged mean flow fields. A total of 100 images were taken at each phase. The Nd-YAG laser, CCD cameras, rotary stage, and BiSlide were precisely synchronized to capture the desired phases of the motion. For every experimental run images from first 2 cycles of the motion were discarded in order to remove transient effects. The PIV laser pulse period and the airfoil motion period were matched with an accuracy of $0.1 \mathrm{~ms}$ for a typical period of approximately $10 \mathrm{~s}$. This produced a slight discrepancy in the model position between the first image and the last image at phases with large speed motion. The maximum shift displacement for all cases was approximately 5 pixels, which corresponds to 0.3 millimeters. In terms of data processing, this discrepancy resulted in an elimination of a datum point near the model surface.

The PIV images were analyzed using an in-house developed MATLAB-based PIV analysis software. The particle displacement is determined in two passes using cross-correlation analysis of displaced interrogation windows. The location of the cross-correlation peak, which gives the particle displacement, is measured with sub-pixel resolution using a Gaussian fit of the cross correlation function around the peak. In the first low-resolution pass a fixed displacement of 6 pixels and an interrogation window of 64 by 64 pixels were used; in the second high-resolution pass the particle displacement measured in the first pass and an interrogation window size of 32 by 32 pixels were used. This corresponds to an approximate spatial resolution of the PIV measurements of $0.64 \mathrm{~mm}$. Several validation criteria were applied to the measured particle displacements. The peak magnitude must be at least three standard deviations above the mean of the cross-correlation function; and the displacement must be within a predetermined range of values in the $\mathrm{x}$ - and $\mathrm{y}$-directions. The range of values in the first pass is fairly large to capture the large range of particle displacements found near the model surface; and small (10 pixels displacement) in the second pass. A median filter is used to find the particle displacement at the points where the PIV validation failed, and to remove outliers. A square grid with 16 pixel spacing was used for all the images which correspond to $250.5 \mathrm{~mm}$ by $167 \mathrm{~mm}$ in physical space.

The median filter used in the PIV post-processing removed outliers based on velocity vector values on spatially adjacent points. To further remove outliers based on large sample-to-sample fluctuations a 3-sigma filter was implemented. First, the sample mean and standard deviation from processed PIV data were calculated at a particular point in the flow field. Then with the sample mean and standard deviation value, each datum point was revisited and the value was compared to \pm 3 standard deviation of the mean value. The datum point was discarded if it lied outside the 3 -sigma region. The highest number of outliers was located in the high shear region and the maximum number of data points removed from the 3-sigma filter was 5 data points for all cases. 


\section{Results}

The airfoil kinematics used in this study is identical to the pure plunge kinematic used by $\mathrm{Ol}$ et $a l .{ }^{17}$ with $k=0.25, S t=0.08$ and $h_{0}=0.5$, which will be defined here as the reference case. The pure plunge kinematic produces a sinusoidal effective angle of attack profile that ranges from $-6^{\circ}$ to $22^{\circ}$ with mean of $8^{\circ}$. As indicated earlier the effective angle of attack profile is defined as the angle of attack for an observer moving with the pivot point of the airfoil motion. The choice of this effective angle of attack is based on the observation that a large leading edge vortex (LEV) and trailing edge vortex (TEV) are present in the flow field. Ol et al. ${ }^{17}$ reported the formation of a LEV and a TEV for the pure plunge motion where the effective angle of attack of the airfoil is well beyond the static stall angle of attack of the SD7003 airfoil at $R e=60,000$. Baik et al. ${ }^{18}$ performed similar experiment using identical conditions with flat plate airfoil, and found that the LEV formation occurs earlier in the phase due to sharper leading edge that influences the point of the separation. Additionally, the effect of different airfoil shape results in similar flow field features when the flow is dominated by a large leading edge separation. The Re effect study on the same pure plunge kinematic was performed by Kang et al. ${ }^{19}$ and it was found that the Re effect is minimal for the cases with large leading edge separation. Based on these recent findings, current study conducted an experiment on a pure plunging flat plate wing at $R e=10,000$.

The general expression for effective angle of attack resulting from harmonically pitching and plunging motion is given by Equation 1 .

$$
\alpha_{\text {eff }}(t)=\alpha_{0}+\alpha_{\text {pitch }}+\alpha_{\text {plunge }}=\alpha_{0}+\theta_{0} \cos (2 \pi f t+\phi)+\arctan (\pi S t \sin (2 \pi f t))
$$

$\alpha_{0}$ is the mean angle of attack, and the phase lag $(\phi)$ between the pitch and plunge motion was set at $90^{\circ}$. The arctangent function causes the effective angle of attack due to the plunge motion to deviate from a sinusoidal profile as $S t$ is increased. The deviation becomes significant at $S t$ approximately greater than 0.15 .

As noted earlier we would like to preserve the sinusoidal effective angle of attack profile for all St. A sinusoidal effective angle of attack produced by the plunge motion was enforced as shown in Equation 2 , where $\alpha_{\text {plmax }}$ is the maximum angle of attack produced by the plunge motion.

$$
\left.\alpha_{\text {eff }}(t)=\alpha_{0}+\theta_{0} \cos (2 \pi f t+\phi)+\alpha_{\text {plmax }} \sin (2 \pi f t)\right)
$$

The plunge motion is unknown and therefore must be obtained by integration of Equation 3 .

$$
\arctan \left(-\frac{h \dot{(t)}}{U_{\infty}}\right)=\alpha_{\text {plmax }} \sin (2 \pi f t)
$$

After some rearrangements, we obtain an ordinary differential equation for the plunge motion which is solved with the requirement that the amplitude of the plunge motion must remain constant in order to preserve the value of $S t$.

$$
\dot{h}(t)=-U_{\infty} \tan \left(\alpha_{\text {plmax }} \sin (2 \pi f t)\right)=h_{0} c \dot{F}(t)
$$


The function $\dot{F}(t)$ in Equation 4 must range between -1 and 1 in order to preserve the amplitude of the motion, and the function must equal to -1 when integrated over a quarter period because the total airfoil displacement equals $-h_{0}$. From these properties, an integral expression for solving $\dot{F}(t)$ can be written, as shown in Equation 5.

$$
\int_{0}^{\frac{1}{4 f}} \dot{F}(t) d t=\int_{0}^{\frac{1}{4 f}}-\frac{U_{\infty}}{h_{0} c} \tan \left(\alpha_{\text {plmax }} \sin (2 \pi f t)\right) d t=-1
$$

$F(t)$ is found numerically through an iterative procedure to determine the constant $\alpha_{\text {plmax }}$. Once $F(t)$ is found, simply multiply the function by the amplitude of the motion to obtain plunge kinematic that preserves the effective angle of attack for a given St. The plunge motion obtained through this approach is no longer sinusoidal. The pitch amplitude, $\left|\theta_{0}\right|$, can be calculated from Equation 2 since the effective angle of attack is kept constant. Table 1 is the list of cases and associated non-dimensional parameters used in current study.

\begin{tabular}{cccccc} 
Case & $S t$ & $k$ & $h_{0}$ & $\left|\theta_{0}\right|\left(^{\circ}\right)\left(\right.$ Sinusoidal $\left.\alpha_{\mathrm{eff}}\right)$ & $\left|\theta_{0}\right|\left(^{\circ}\right)$ (Sinusoidal motion) \\
\hline A & 0.08 & 0.250 & 0.500 & 0.00 & 0.00 \\
1 & 0.10 & 0.314 & 0.500 & 3.56 & 3.39 \\
2 & 0.15 & 0.471 & 0.500 & 11.69 & 11.18 \\
3 & 0.20 & 0.628 & 0.500 & 19.14 & 18.09 \\
4 & 0.15 & 0.628 & 0.375 & 11.69 & 11.18 \\
5 & 0.10 & 0.628 & 0.250 & 3.56 & 3.39 \\
6 & 0.10 & 0.419 & 0.375 & 3.56 & 3.39
\end{tabular}

Table 1. List of cases and associated non-dimensional parameters.

Case A is the effective angle of attack profile from the pure plunge kinematic presented by $\mathrm{Ol}$ et al. ${ }^{17}$. Cases 1 through 6 are the new cases derived for the same effective angle of attack profile. These cases were chosen to keep $S t, k$, or $h_{0}$ constant; these cases will distinguish the effect of a non-dimensional parameter while preserving the effective angle of attack. As $S t$ is increased, $\left|\theta_{0}\right|$ also increased in order to compensate for the increase in effective angle of attack due to the plunge motion. Note that the pitch amplitude is only a function of St. The difference in the pitch amplitude between prescribed sinusoidal motion and sinusoidal effective angle of attack is also shown in Table 1. The difference in the effective angle of attack profile between prescribed sinusoidal effective angle of attack and sinusoidal plunge motion for $S t=0.2$ is highlighted in Figure 2 . 


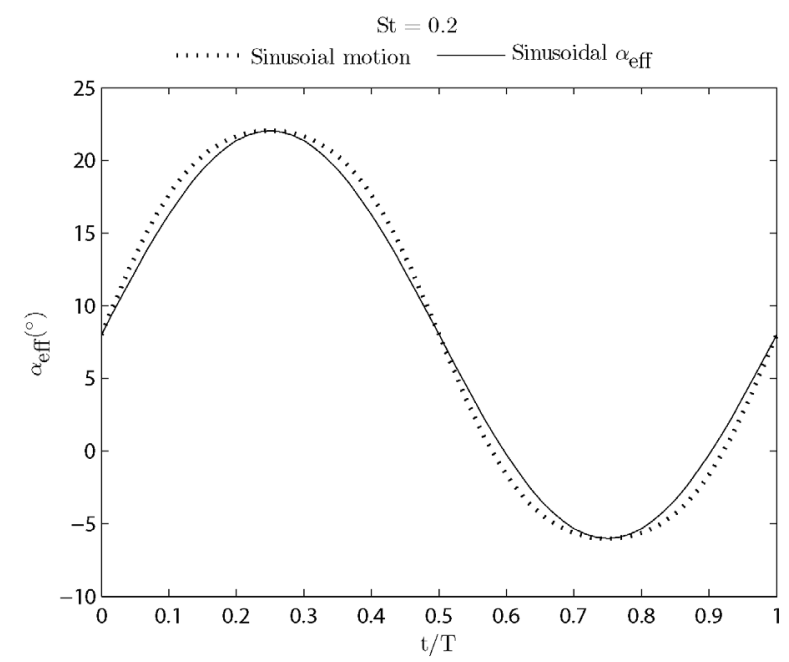

Figure 2. Different effective angle of attack profile produced by sinusoidal plunge motion.

The difference is subtle in the actual plunge motion but the resulting effective angle of attack profile is different. A further increase in $S t$ for sinusoidal plunge motion will cause the effective angle of attack to have dual peaks when $S t$ is greater than 0.3.

All the cases shown in Table 1 can be summarized in the $k-h_{0}$ space as shown in Figure 3 . The red lines denote the facility limits; the vertical line at $h_{0}=0.5$ is the limit introduced by the water channel width and curved red line is the limit introduced by the maximum pitch acceleration of the step motor.

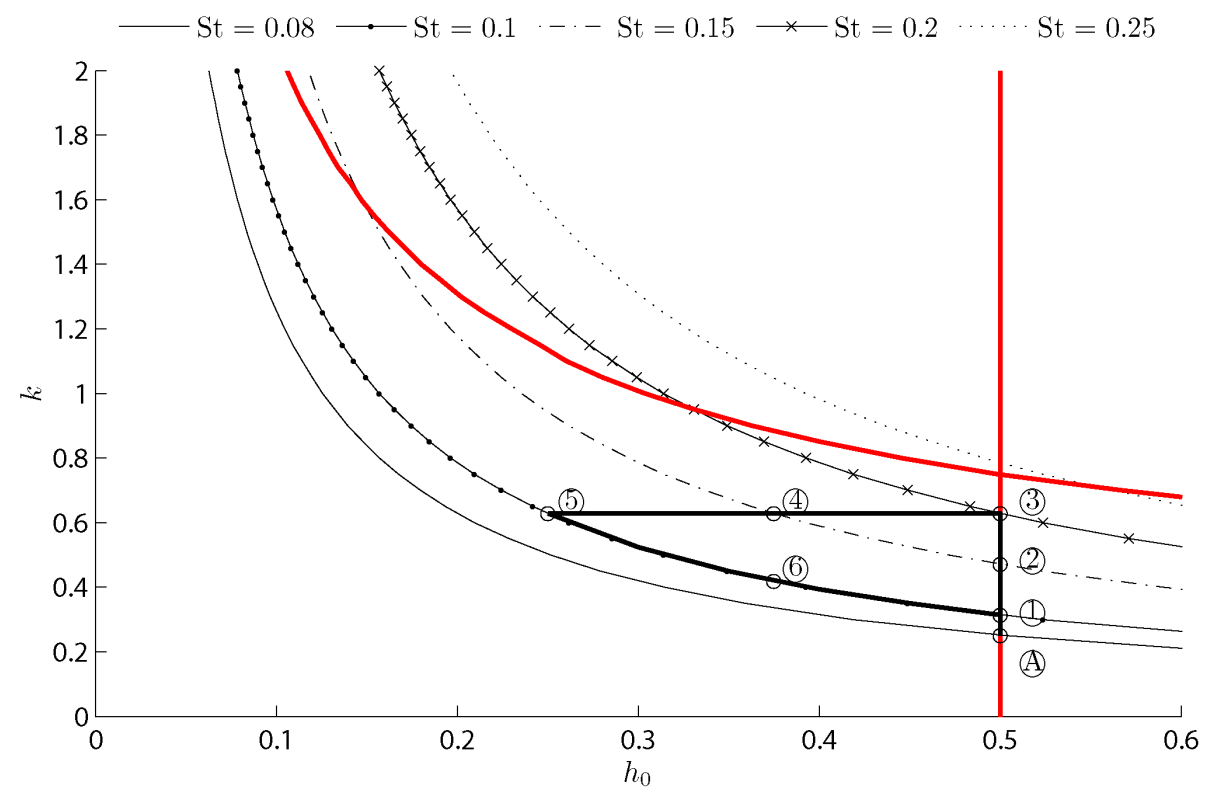

Figure 3. Cases 1 through 6 cover constant $h_{0}$, constant $k$, and constant $S t$ regimes for constant effective angle of attack.

There are 3 regimes of interest; constant $h_{0}$ (Case $1 \sim 3$ ), constant $k$ (Case $3 \sim 5$ ), and constant St (Case 1,6, and 5). Additional comparison can be made between cases 2 and 4 as the $S t$ is same for both at 0.15 . A qualitative and quantitative analysis of flow field around the airfoil using dye flow visualization and phase-averaged PIV will be made for the 3 regimes. 


\section{A. Constant $h_{0}$}

Constant $h_{0}$ space covers cases 1,2 , and 3; St is varied from 0.1 to 0.2 by increasing reduced frequency from 0.314 to 0.628 . Figures A1 A3 (Appendix A) provides a summary of qualitative analysis of instantaneous dye flow visualization, and phased-averaged velocity and vorticity contours obtained from PIV. The agreement between dye flow visualization and PIV data is excellent for all 3 cases. There are notable similarities in the vorticity field for these 3 cases which are shown in Figure B1 (Appendix B). A pair of small vortices is observed at phase $30^{\circ}$ for all 3 cases which then develops into a single large leading edge vortex (LEV) in subsequent phases. A notable discrepancy at constant $h_{0}$ regime is the rate at which the LEV develops. Case 1, which has the lowest $k$ value of 0.314 develops the LEV more rapidly than cases with higher $k$. The vorticity contour of case 1 at phase $90^{\circ}$ is in good qualitative agreement with phase $120^{\circ}$ of case 2 and phase $150^{\circ}$ of case 3 . This finding is consistent with the numerical results obtained by Visbal et al. ${ }^{20}$ who reported an increase in lag between the formation of LEV and the pitching motion with increasing $k$. Another interesting phenomenon is the formation of TEV as the LEV encounters the trailing edge of the airfoil. The TEV is smaller in size compared to the LEV and it is observed at phase $150^{\circ}, 210^{\circ}$, and $240^{\circ}$ for cases 1,2 , and 3 , respectively. The delay in formation of the TEV is directly related to the formation time of the LEV. The dye flow visualization shows the TEV forming at latter phases for cases 2 and 3; fully developed TEV is formed away from the trailing edge of the airfoil as $k$ is increased.

A quantitative approach was taken to highlight the similarities and differences of the constant $h_{0}$ regime. The comparison of $\mathrm{x}$-component velocity profiles normal the airfoil surface at the quarter-chord location for cases 1, 2, and 3 is shown in Figure 4.

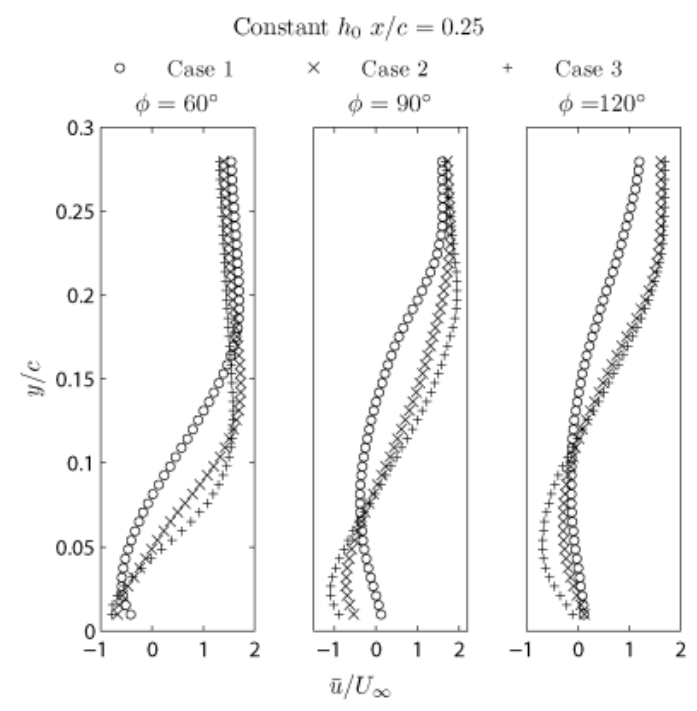

(a)

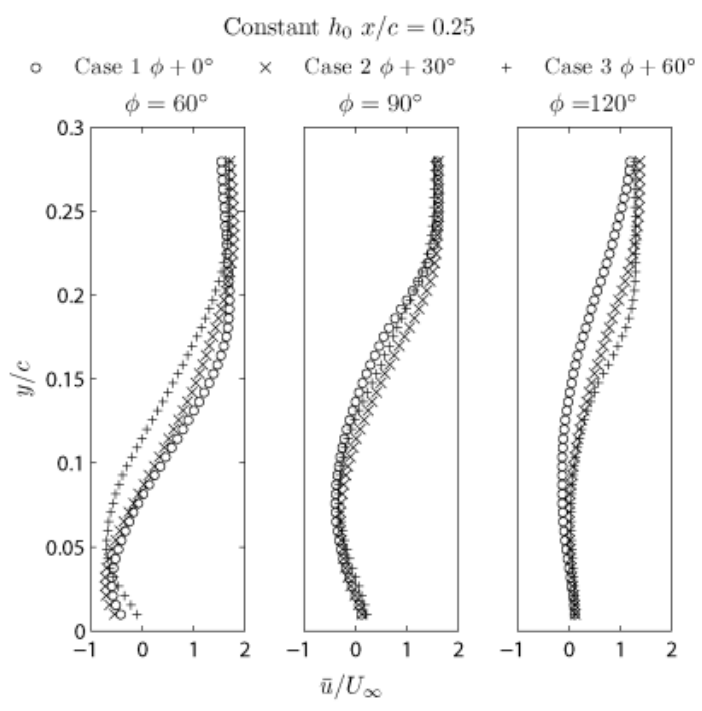

(b)

Figure 4. The $\mathrm{x}$-component velocity profile comparison at $x / c=0.25$ for constant $h_{0}$ regime without phase-shift (a), and with phase-shift (b).

Figure $4 \mathrm{a}$ shows the phase-locked comparison and Figure $4 \mathrm{~b}$ is the phase-shifted comparison of velocity profiles. The apparent delay in the formation of LEV is illustrated in Figure $4 \mathrm{a}$. Case 1 has a larger separated region at phase $60^{\circ}$ but the magnitude of the reverse flow is larger for cases 2 and 3 because the 
vortex is 'tighter' at the initial stage of formation. Similar observation is made at phase $90^{\circ}$; case 1 has the largest reverse flow region and case 3 has the highest magnitude of reverse flow. In order to assess the duration of the delay present between the cases, Figure $4 \mathrm{~b}$ plots case 2 and 3 data with phase shift of $30^{\circ}$ and $60^{\circ}$, respectively. At phase $60^{\circ}$ of case 1 , cases 1 and 2 are in good agreement but the case 3 velocity profile shows a more developed vortical structure, evidenced by the larger separated region. This suggests that the phase delay between cases 1 and 3 is less than $60^{\circ}$. The quantitative agreement is best at phase $90^{\circ}$ of case 1 , and this was also observed from the vorticity contours.

\section{B. Constant St}

Constant $S t$ regime refers to cases 1,6 , and $5 ; S t$ is held constant at 0.1 with varying combination of $k$ and $h_{0}$. Figures A1, A5, and A6 provides a summary of instantaneous dye flow visualization, and phase-averaged velocity and vorticity contours obtained from PIV. Figure B2 summarizes the vorticity contours obtained from PIV. The qualitative analysis is similar to the constant $h_{0}$ regime; a pair of vortices forming at the leading edge, the formation of a large LEV, shedding of small vortical structures from the leading edge, and delayed formation of the LEV due to increase in $k$. This indicates that the delayed LEV formation is an effect of reduced frequency $k$, not St. Even the phase at which the TEV is captured is similar to the constant $h_{0}$ regime due to the similar delay in formation of the LEV. An interesting observation can be made regarding the formation of TEV. For cases 1 and 6 , the airfoil is in the downstroke motion as the LEV passes through the trailing edge of the airfoil. However, the airfoil is in the upstroke motion for case 5. As the airfoil trailing edge interacts with the evincing LEV, it leaves no room for the TEV to form. The TEV is forced to form when the LEV is sufficiently far away from the trailing edge and the resulting location of fully developed TEV is downstream of the trailing edge of the airfoil for case 5.

Figure 5 plots x-component velocity profiles at the quarter-chord location for the constant $S t$ regime.

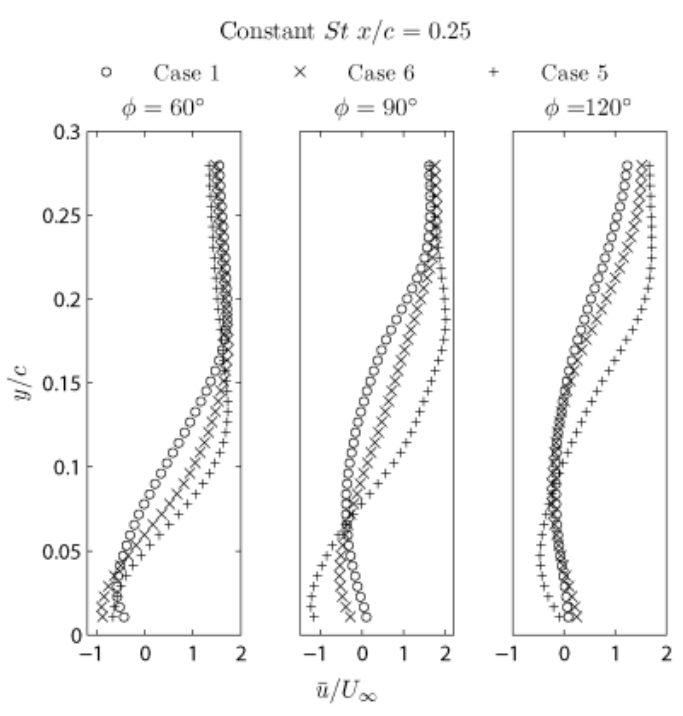

(a)

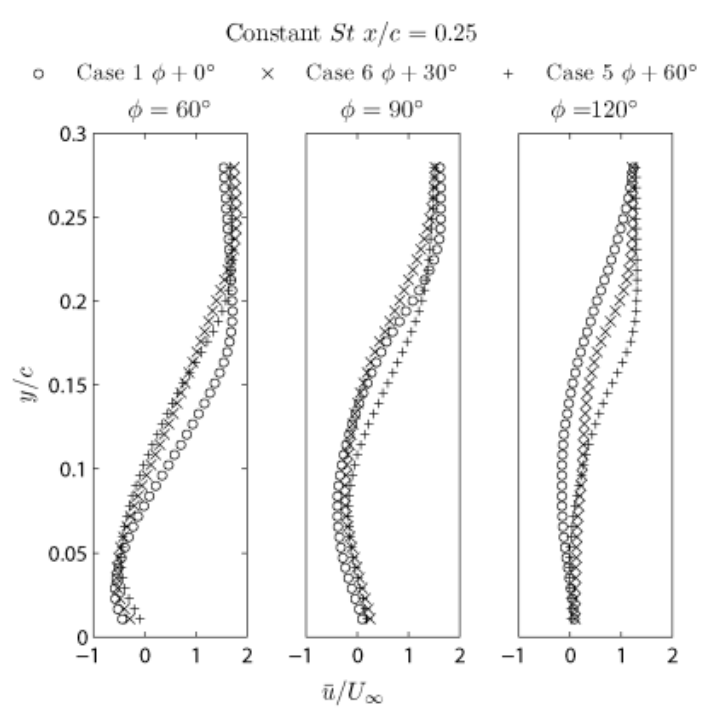

(b)

Figure 5. The $\mathrm{x}$-component velocity profile comparison at $x / c=0.25$ for constant $S t$ regime without phase-shift (a), and with phase-shift (b). 
Figure 5a shows the phase-locked comparison and Figure 5b is the phase-shifted comparison of velocity profiles. It is not surprising that the comparison is similar to the constant $h_{0}$ regime. The phase-locked comparison shows case 1 having the largest separation at earlier phases and case 5 showing a sign of slowest development of separated region. The agreement is improved when cases 6 and 5 were phase shifted by $30^{\circ}$ and $60^{\circ}$, respectively.

\section{Constant k}

Constant $k$ regime refers to cases 3,4 , and $5 ; k$ is held constant at 0.628 with varying $h_{0}$ to produce $S t$ ranging from 0.1 to 0.2 . Figures A3 A5 provides a summary of qualitative analysis of dye flow visualization, velocity and vorticity contours obtained from PIV. A key observation from the constant $h_{0}$ and $S t$ regimes was that the $k$ drives the delayed formation of the LEV. Thus, the expectation for constant $k$ regime is that the formation time of LEV is the same for cases 3, 4, and 5. Figure B3 supports this hypothesis by showing excellent qualitative agreement of vorticity contours and streamlines for all 3 cases. The size and the location of the LEV are the same for all 3 cases and the location of TEV and its strength are also in excellent agreement. The only discrepancy present is found at phase $60^{\circ}$ of case 5 where a pair of vortices is more distinct than in the other 2 cases. However, this vortex pair forms a single large LEV at a subsequent phase and the discrepancy between all 3 cases is no longer present.

Further comparison of $\mathrm{x}$-component velocity profiles normal to the airfoil surface located at the quarter-chord point are shown in Figure 6. The quantitative agreement between all 3 cases at all phases is good overall. All 3 cases develop a strong but narrow reverse flow region at phase $60^{\circ}$ which then thickens as the LEV grows in size and the peak velocity reaches 2 times the free stream velocity at phase $90^{\circ}$. Phases $120^{\circ}$ through $180^{\circ}$ indicate the possibility of detached LEV because the velocity gradient changes from being steep to shallow near the wall. At phase $180^{\circ}$, the reverse flow region is almost diminished and the flow reattaches in subsequent phases. The agreement is worst at phase $120^{\circ}$, especially in the velocity profile for case 4 . From the vorticity contours shown in Figure B3, case 4 phase $120^{\circ}$ shows a secondary vortex that resides near the quarter-chord location next to the LEV. The shedding of small vortical structures from the leading edge is a general feature observed in all the cases. The sudden deceleration of the $\mathrm{x}$-component velocity profile between $y / c=0.15$ and 0.25 for case 4 phase $120^{\circ}$ is influenced by the presence of this small vortex, and the agreement with cases 3 and 5 is poor. Additionally, the velocity gradient normal to the airfoil at the quarter-chord location is greatest at phase $120^{\circ}$ and therefore the number of valid data points at this phase was smaller than for other phases. Nonetheless, the quantitative agreement is good at other phases and it is a strong indication that constant $k$ results in similar flow field with respect to the airfoil regardless of $S t$ and $h_{0}$ values.

As the $S t$ increases from 0.1 to 0.2 , the pitch amplitude must increase with $S t$ in order to preserve the effective angle of attack profile. The pitch amplitude is the greatest for case 3 with $\left|\theta_{0}\right|=19.14^{\circ}$, and lowest for case 5 with $\left|\theta_{0}\right|=3.56^{\circ}$. This provides a range of maximum pitch rate that varies from $10.57 \%$ to $1.97 \%$ s. Therefore, it is necessary to perform an additional comparison at chord location other than the quarter-chord location since the comparison done at the quarter-chord location neglects the effect of pitch rate. The x-component velocity profile comparison at $x / c=0.75$ for the constant $k$ is shown in Figure 7 . 

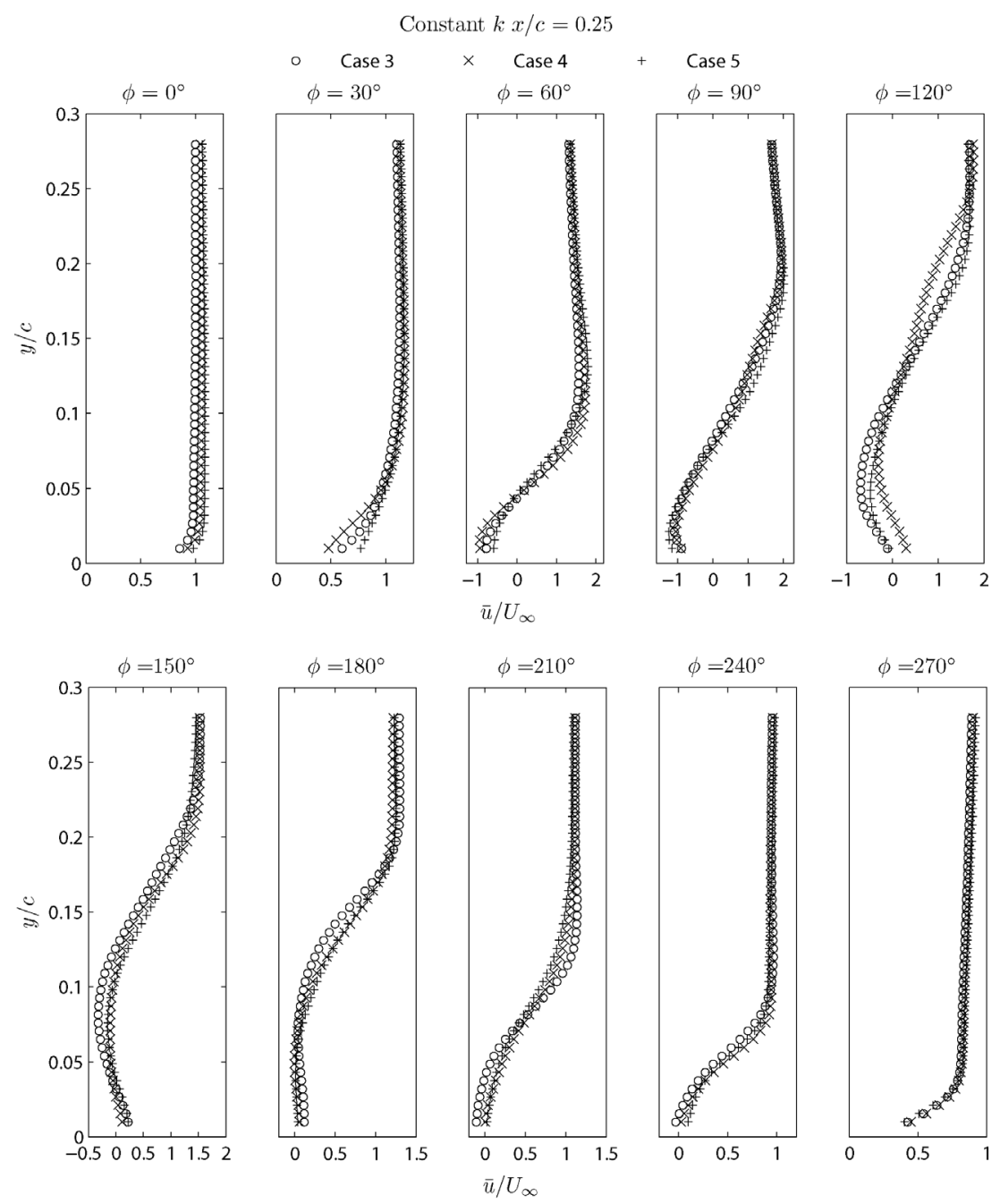

Figure 6. The $x$-component velocity profile comparison at $x / c=0.25$ for constant $k$ regime.

Again, the quantitative agreement of velocity profiles for the 3 cases is good overall. Some discrepancies are observed at latter phases during the upstroke motion. Initially, case 3 shows stronger reverse flow region than the other cases at phase $210^{\circ}$, but case 5 is in disagreement with the rest at phase $240^{\circ}$. The LEV resides near the trailing edge of the airfoil and the interaction between the LEV and the location of TEV may influence the velocity profiles at $x / c=0.75$ differently. Nonetheless, a quantitative agreement of velocity profiles at $x / c=0.75$ suggests 2 things: 1 ) the pivot point effect is negligible for the pitch rates used in this study, and 2) the constant effective angle of attack profile produces consistent flow field normal to the airfoil. This implies an interesting conclusion regarding the effect of pitch. Assuming that the force history with respect to the airfoil is the same for constant $k$ regime, the roll of pitch is to redirect the direction of the force rather than affecting its magnitude. Higher $S t$ induces higher pitch amplitude for a constant effective angle of attack profile, and this may explain why certain range of $S t$ is desirable; it is possible to redirect drag to produce thrust by increasing St. However, agreement in instantaneous velocity field does not necessarily imply same force time history because the time rate of change of the velocity field is crucial in computing the force in unsteady aerodynamics. Additionally, a 
theoretical approach such as Theodorsen's linear theory suggests that the force exerted on the airfoil is different for varying pitch rates ${ }^{21}$.
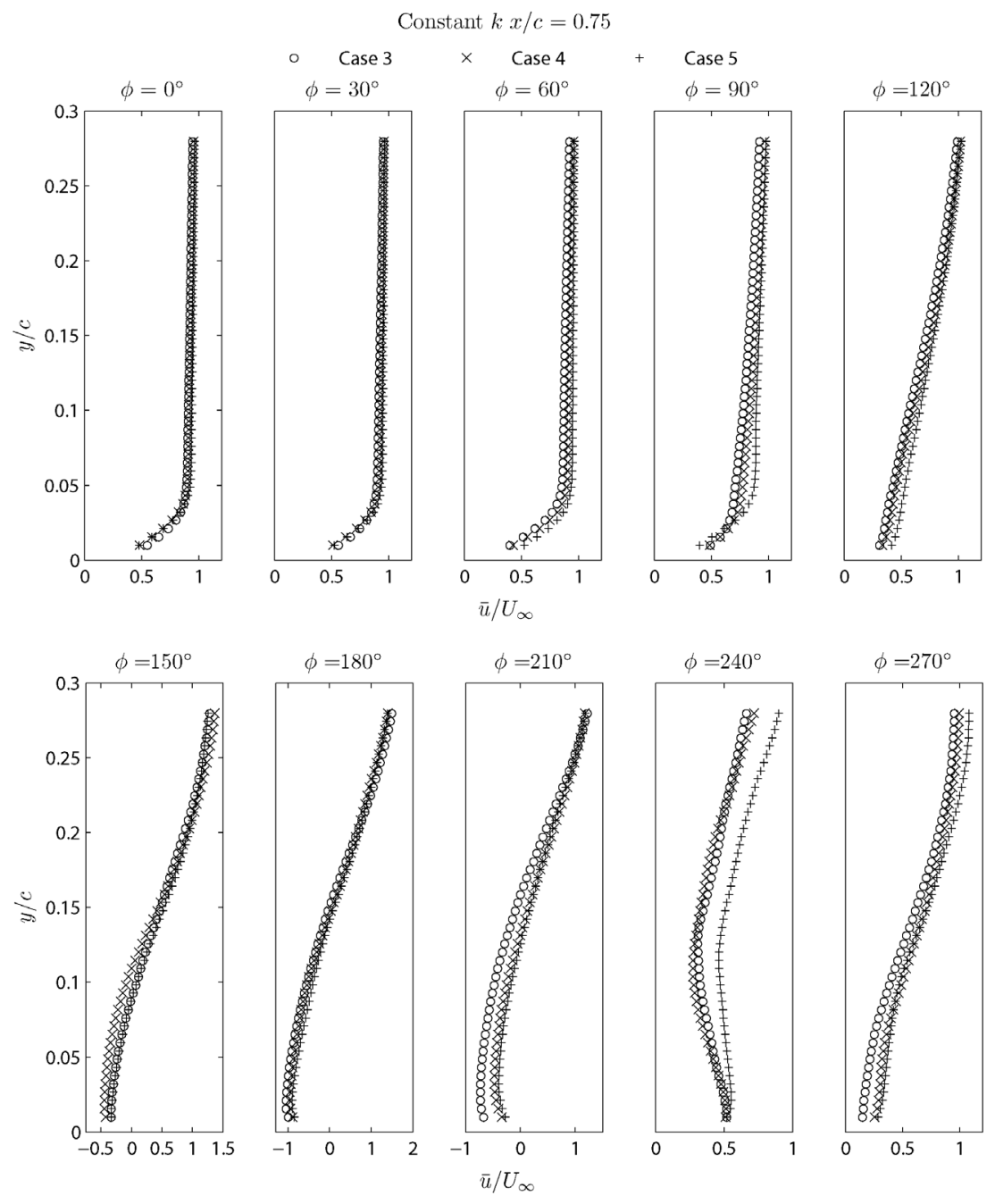

Figure 7. The $x$-component velocity profile comparison at $x / c=0.75$ for constant $k$ regime.

\section{The Leading Edge Vortex}

The effective angle of attack profile used in this study results in a large leading edge separation and the formation of a LEV and a TEV. As the LEV evince downstream, small vortical structures are formed from the leading edge along the high shear region. It is observed that the frequency of the vortices being shed is in a good agreement for all cases; the instability in the shear layer may be amplified and cause these small vortical structures to be formed. The amplification factor in a shear layer is directly related to the ratio of momentum thickness and the free stream velocity ${ }^{22}$. The constant $R e$ preserves the magnitude of the free stream velocity, and identical effective angle of attack profile helps maintain the separation point at the leading edge to be the same. This will cause the momentum thickness at the point 
of separation to be the same for all cases hence inducing similar shedding frequency of instability vortices in the shear layer.

Observing the similarity in the velocity profiles around the airfoil, we further probe the location and strength of the leading edge vortices for the different cases. Two different methods were used to locate the LEV core: Q-criterion introduced by Jeong et al. ${ }^{23}$ and local streamline patterns with respect to the airfoil. The value of local maximum $Q$ was used to determine the location of the LEV core. The LEV core locations calculated from both methods is collapsed into a single plot with respect to the airfoil and it is shown in Figure 8.

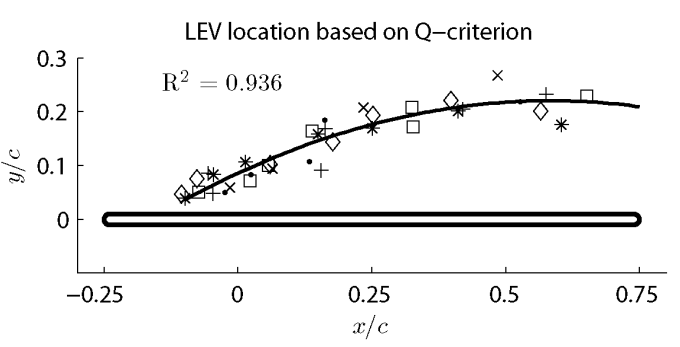

(a)

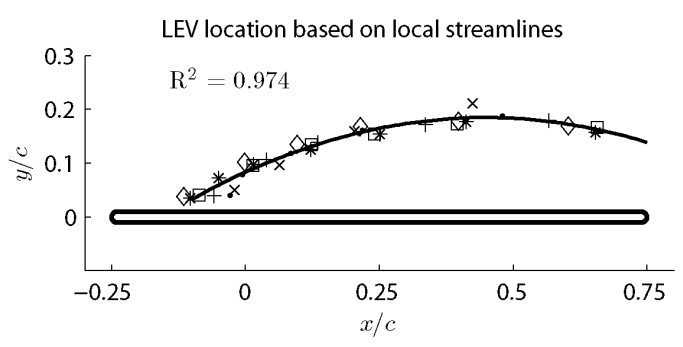

(b)

Figure 8. The LEV trajectory exists based on Q-criterion (a) and local streamlines (b).

Both methods indicate the trajectory of LEV core with respect to the airfoil. Note that Figure 8 does not include any temporal information; the LEV core locations occur at different phase for different cases but the trajectory is independent of any phase information and the orientation of the airfoil. The LEV core locations based on local streamline patterns fared better than Q-criterion. The scatter of the LEV location using the Q-criterion can be explained by the structure of the LEV. The Q-contours of the LEV occasionally showed dual peaks which may suggest that those small vortices shed from the leading edge coalesce with the LEV, and it is illustrated in Figure 9. Note that the small vortical structures in the shear layer have a value of Q comparable to the LEV core, and it will be interesting to calculate how much they contribute to the overall strength of the LEV.

A $2^{\text {nd }}$ order polynomial was curve fitted through LEV core locations in order to obtain a quantitative understanding of the trend shown in Figure 8. The order of polynomial was chosen with no significance but higher order polynomial did result in stronger correlation. The correlation coefficient fared better for LEV core location based on local streamline patterns. Strong correlation coefficients for both methods indicate that LEV core trajectory is dictated by the effective angle of attack profile; all the cases have different non-dimensional parameter governing pitching and plunging airfoil including the pitch rate. The only other parameter that is shared by all the cases is the $R e$. The $R e$ effect on the trajectory of the LEV core would complete the assessment of this analysis.

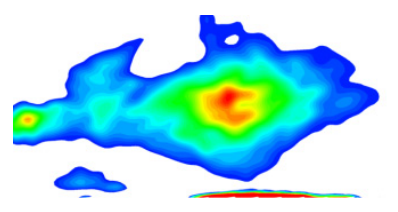

(a)

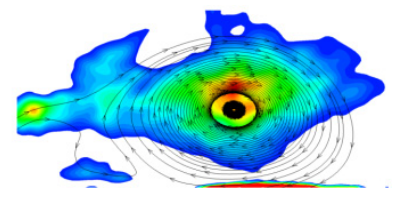

(b)

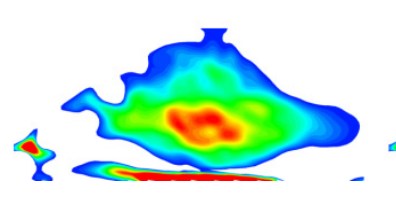

(c)

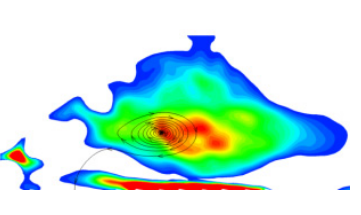

(d)

Figure 9. LEV with a single Q-value peak (a), and associated streamline pattern (b). LEV with multiple Q-value peaks (c), and associated streamline pattern (d). 
The LEV develops in size and strength along the set trajectory with respect to the airfoil, and the simplest approach to estimate the strength of the LEV is to compute its circulation. The flow field is nominally $2 \mathrm{D}$ and therefore the circulation can be computed by the summation of z-vorticity values within the LEV. While there is no systematic way to characterize the size of the LEV, one way to characterize its size is to consider the value of vorticity in the flow field. The circulation can be computed by accounting for vorticity value that is above a certain threshold value in the flow field. The threshold value was chosen arbitrarily but this value was applied for all cases. The threshold value of normalized vorticity used in this study was 8 . After the filtering process, the width of the LEV was chosen manually and only the negative values of vorticity were considered for circulation calculation. Figure 10 illustrates the filtering process and the resulting size of the LEV.

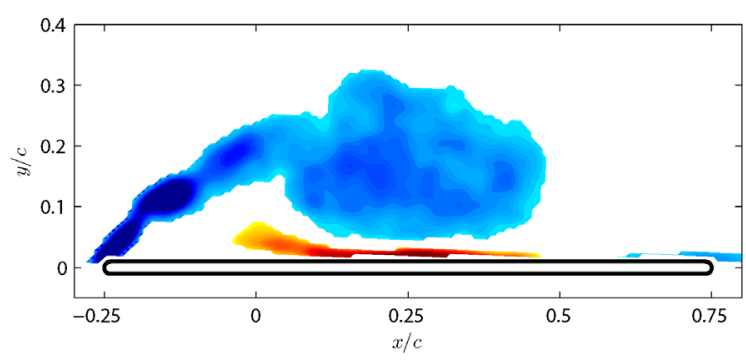

(a)

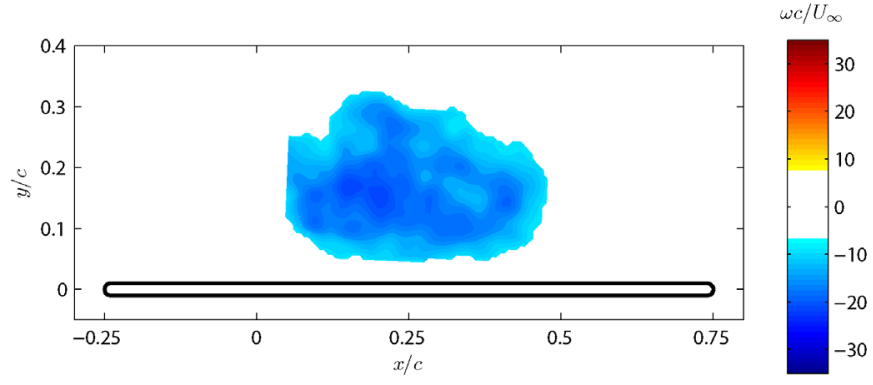

(b)

Figure 10. Normalized vorticity contour above the threshold value for the entire flow field (a), and the LEV (b).

The calculated circulation value of the LEV for all cases were plotted with respect to the airfoil using the LEV position obtained from the Q-criterion and local streamline patterns. Figure 11 summarizes the LEV circulation results.

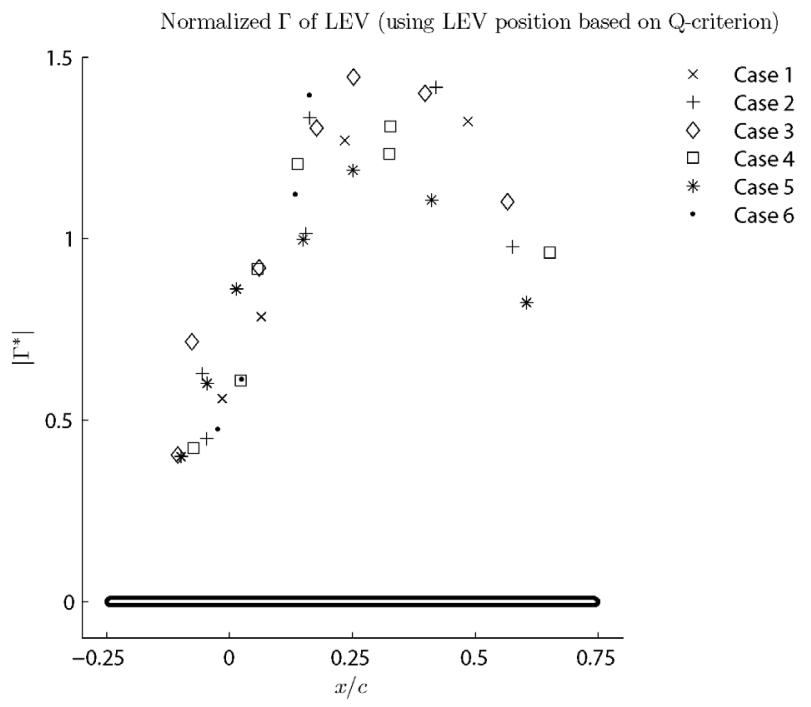

(a)

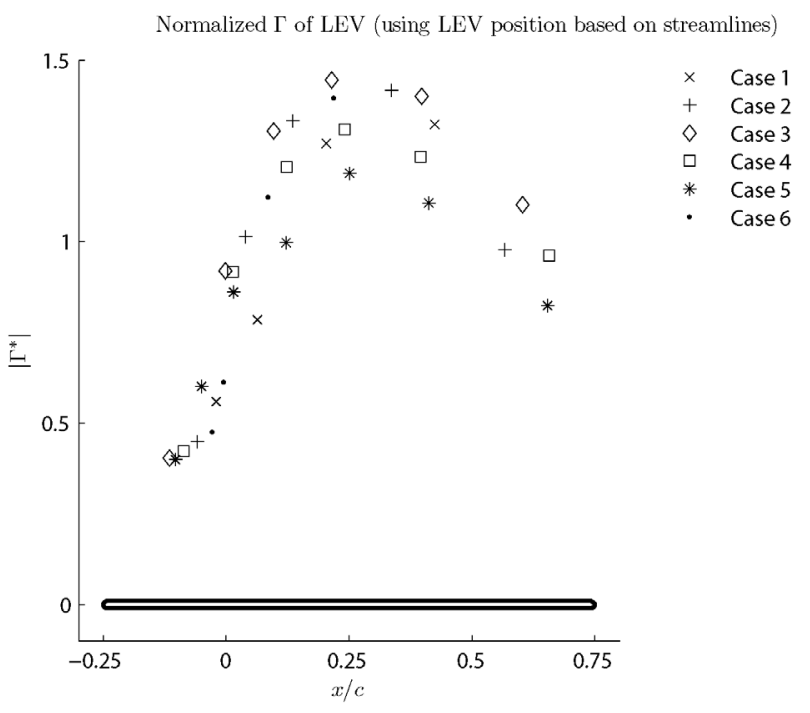

(b)

Figure 11. Normalized circulation of the LEV. The LEV position based on maximum Q-value (a), and local stream patterns (b). 
There is an observable trend in the LEV circulation. The LEV starts to form $x / c \approx 0.15$ and it grows in strength until it reaches the maximum circulation at $x / c \approx 0.5$. A decrease in circulation past $x / c \approx 0.5$ suggests that the LEV may have detached and it is dissipating while convecting downstream. Note that the discrepancies in the location of the LEV between the two methods used do not affect the circulation of the LEV. The circulation of LEV is only sensitive to the assigned vorticity threshold value, and the overall trend was similar to Figure 11 when the threshold value was altered. Likewise to the LEV trajectory, the circulation is independent of $S t, k$, and $h_{0}$; the $R e$ and effective angle of attack profile are the governing parameters responsible for the trend observed in the LEV circulation.

\section{Conclusions}

An experimental study of the effect of parameters governing pitching and plunging airfoils at $R e$ $=10,000$ is presented in this paper. The effective angle of attack profile was kept constant and the $S t, k$, and $h_{0}$ were varied. The plunge motion of the airfoil had to be modified for a sinusoidal effective angle of attack profile, and the plunge motion was no longer sinusoidal after the modification. A dye flow visualization and 2D phase-averaged PIV techniques were used to qualitatively and quantitatively assess the flow field around the pitching and plunging flat plate. Notable conclusions include:

- Increase in reduced frequency results in delayed formation of LEV; constant $S t$ and $h_{0}$ regimes both showed delayed LEV formation as $k$ was increased. The $\mathrm{x}$-component velocity profile comparison qualitatively highlighted the presence of the delay but the exact delay value could not be extracted.

- The flow with respect to the airfoil is the same for the cases with same $k$ and different $S t$ and $h_{0}$. The comparison of $\mathrm{x}$-component velocity at phases ranging from $0^{\circ}$ to $270^{\circ}$ showed good quantitative agreement. A constant effective angle of attack profile produces almost identical flow field normal to the airfoil for the same reduced frequency, $k$.

- The pitch rate and pivot effect were negligible in the cases presented. The pitch rate varied from $1.97 \%$ to $10.57 \%$ with pivot point at the quarter-chord location. The x-component velocity profile at $x / c=0.75$ showed excellent agreement which suggests that the pivot effect is negligible for the pitch rate considered in this study.

- Small vortical structures are formed in the high shear region created by leading edge separation due to the instability of the shear layer. The frequency of these vortical structures is same in all cases. The Q-contours of the LEV occasionally showed multiple peaks which suggest that these small vortices shed from the leading edge coalesce into the LEV.

- There exists the LEV trajectory with respect to the airfoil; the effective angle of attack profile determines this trajectory as $S t, k, h_{0}$, and $\theta_{0}$ were varied for different cases. Both Q-criterion and local streamline patterns were able to capture the trend in LEV core path with acceptable correlation of data points. 
- The circulation of LEV is independent of $S t, k$, and $h_{0}$; the effective angle of attack profile and $R e$ is responsible for the observable trend in the LEV circulation. The LEV starts to form $x / c \approx 0.15$ and it grows in strength until it reaches the maximum circulation at $x / c \approx 0.5$. A decrease in circulation past $x / c \approx 0.5$ suggests that the LEV may have detached and it is dissipating while convecting downstream.

\section{Acknowledgments}

This work has been supported in part by the Air Force Office of Scientific Research's Multidisciplinary University Research Initiative (MURI) and by the Michigan/AFRL (Air Force Research Laboratory)/Boeing Collaborative Center in Aeronautical Sciences. 


\section{Appendix A}

\section{Case 1}

$\left(k=0.314, S t=0.10, h_{0}=0.5\right)$

Phase Flow Visualization $\quad \bar{u} / U_{\infty} \quad \omega c / U_{\infty}$

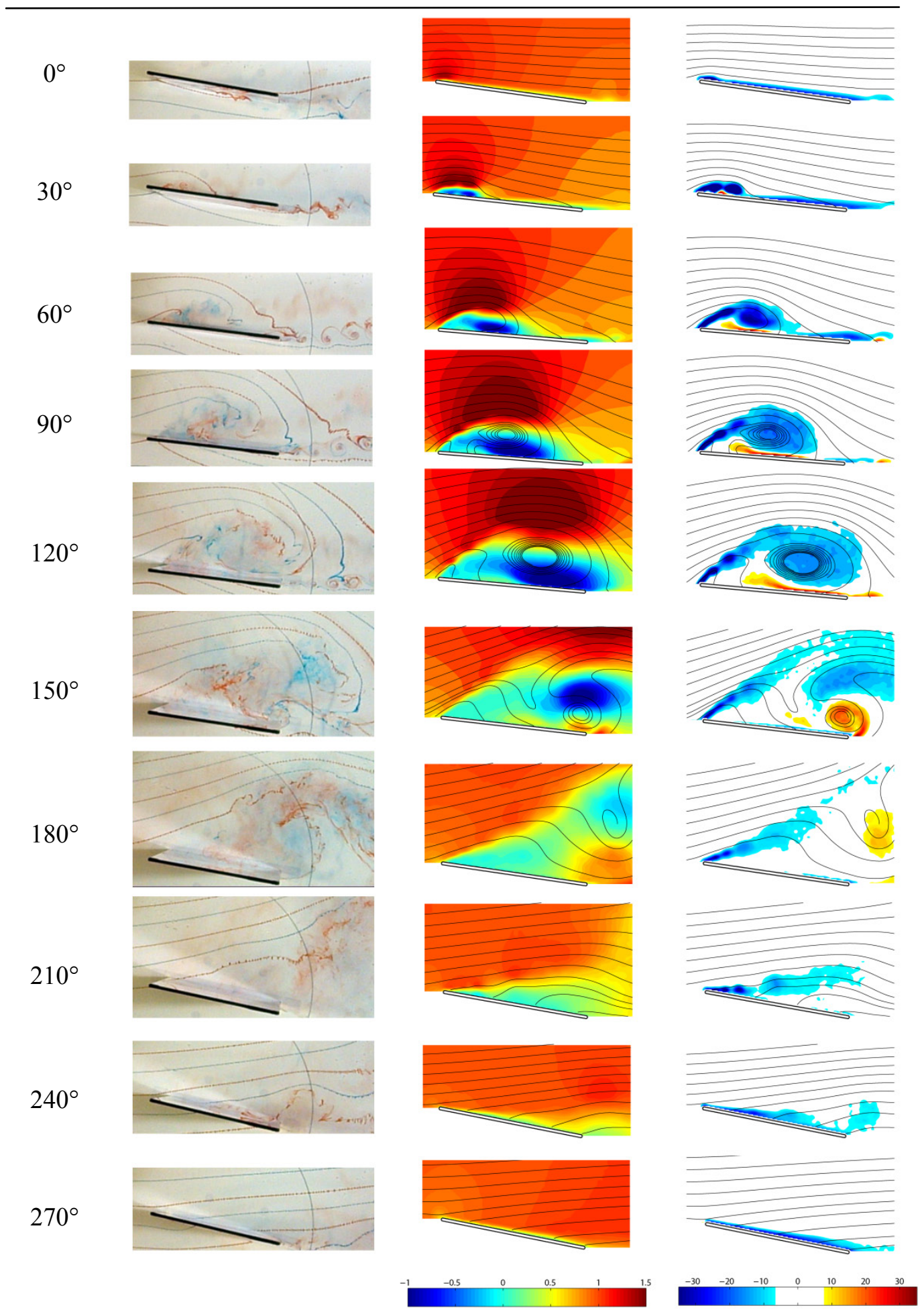

Figure A1 
Case 2

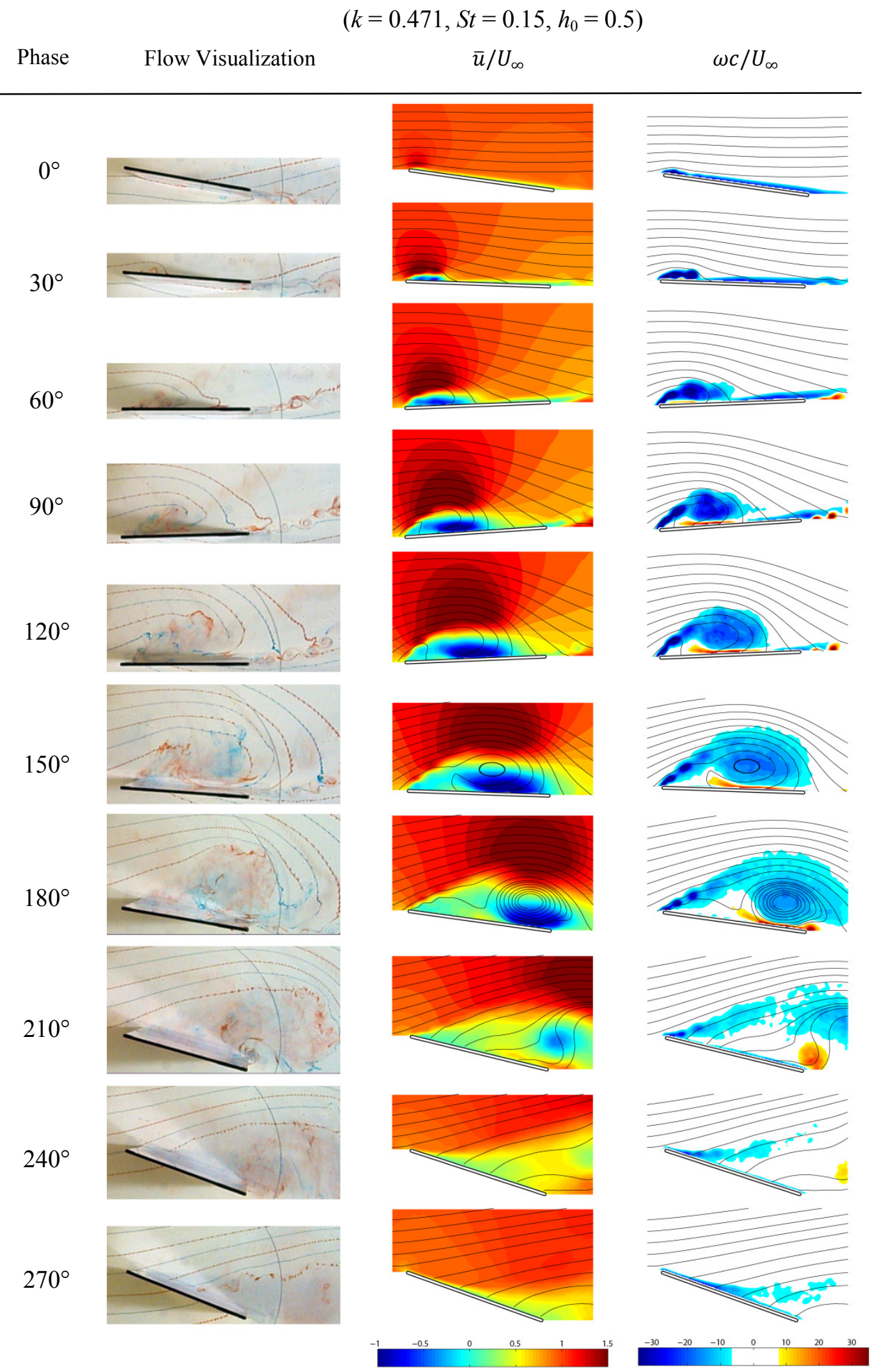

Figure A2 
Case 3

$\left(k=0.628, S t=0.20, h_{0}=0.5\right)$

Phase

Flow Visualization

$\bar{u} / U_{\infty}$

$\omega c / U_{\infty}$

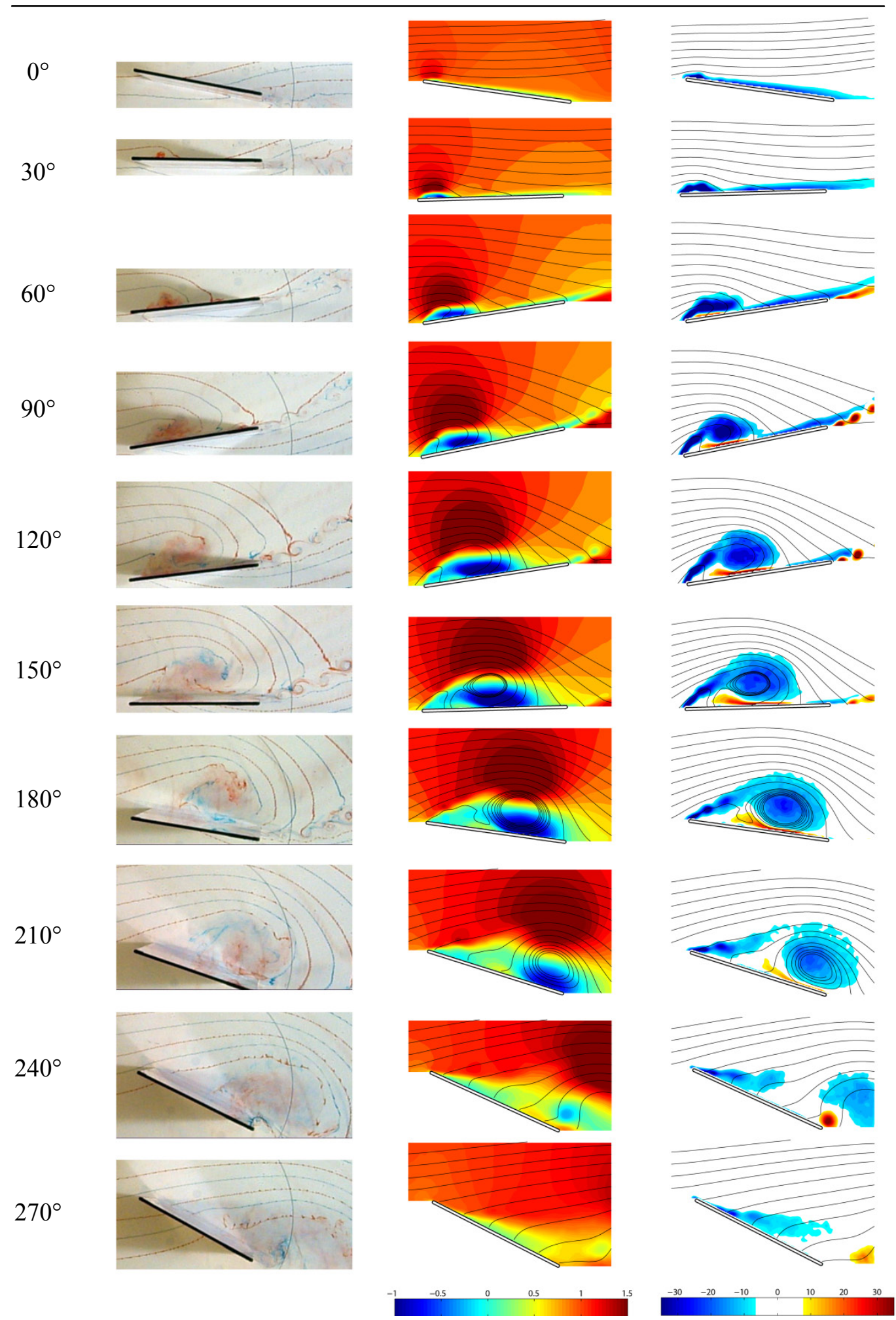

Figure A3 
Case 4

$$
\left(k=0.628, S t=0.15, h_{0}=0.375\right)
$$

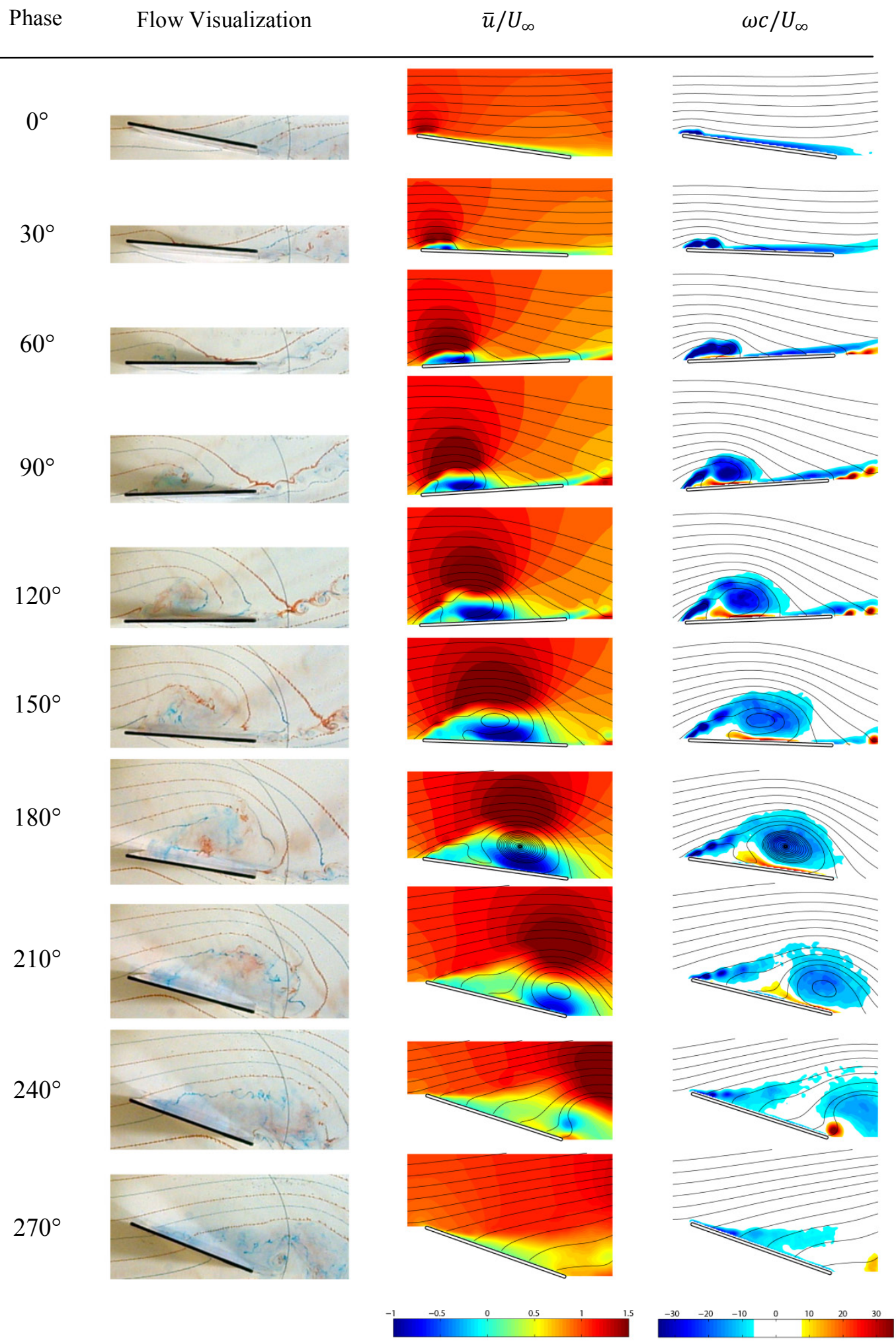

Figure A4 
Case 5

$\left(k=0.628, S t=0.10, h_{0}=0.250\right)$

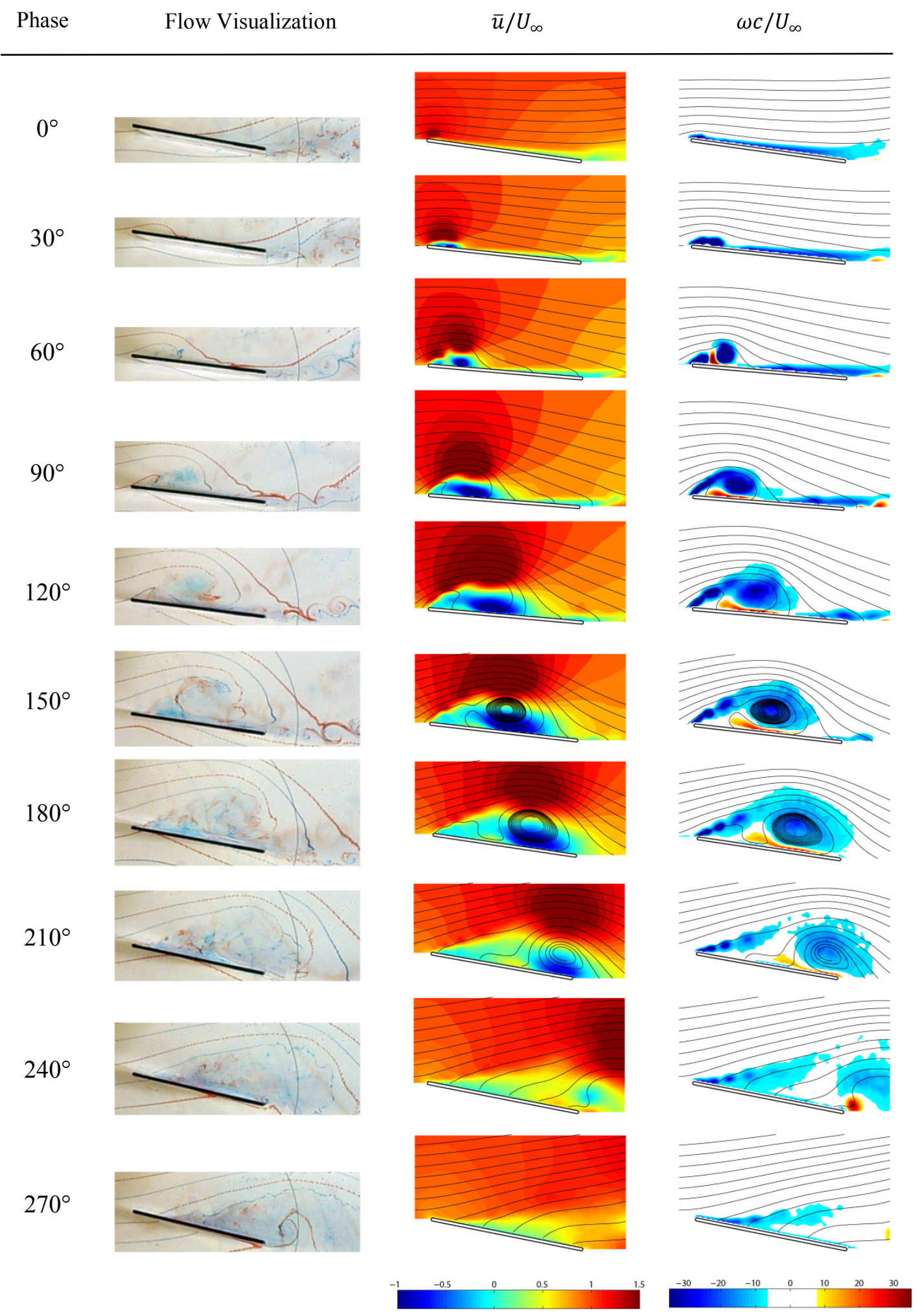

Figure A5 
Case 6

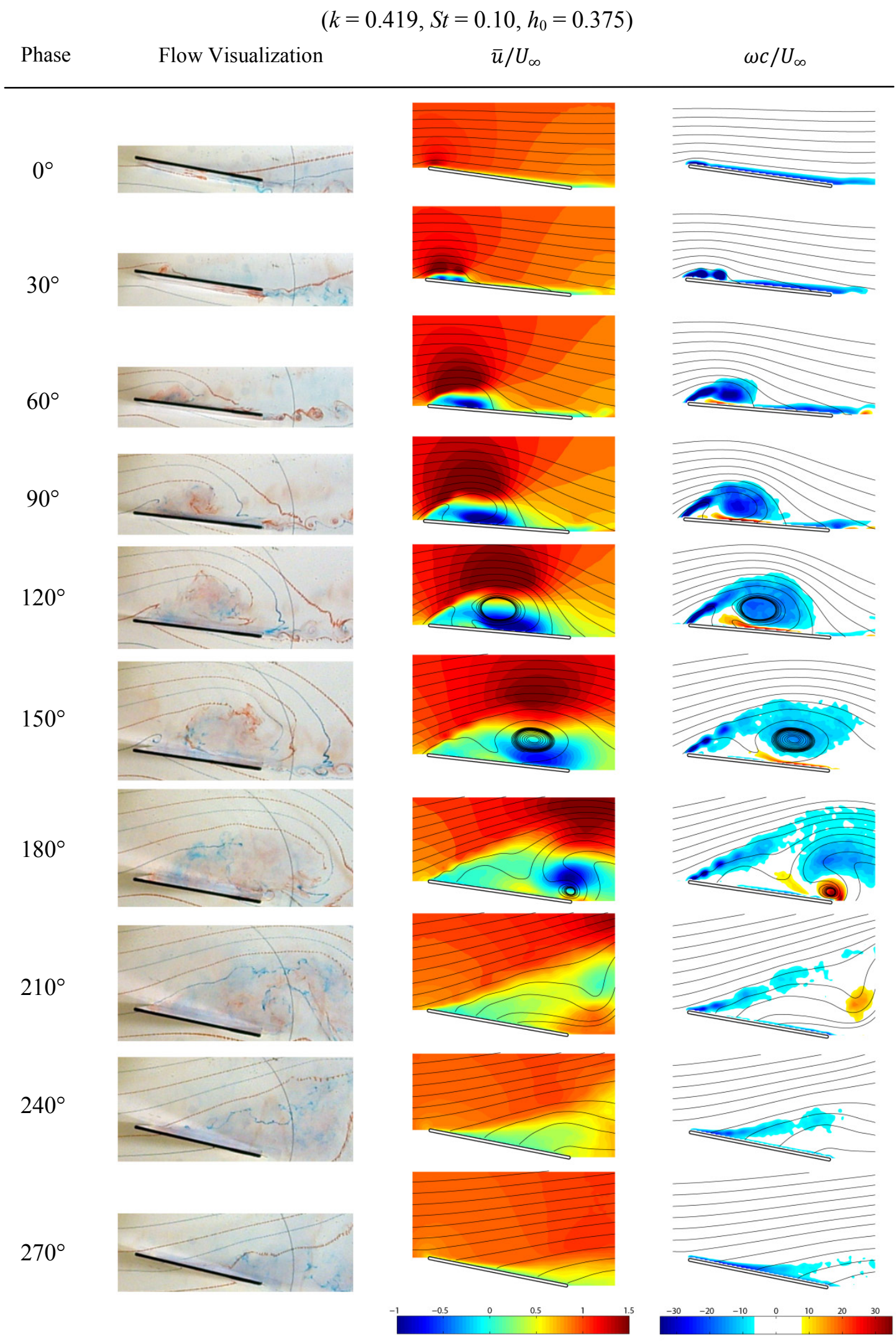

Figure A6 


\section{Appendix B}

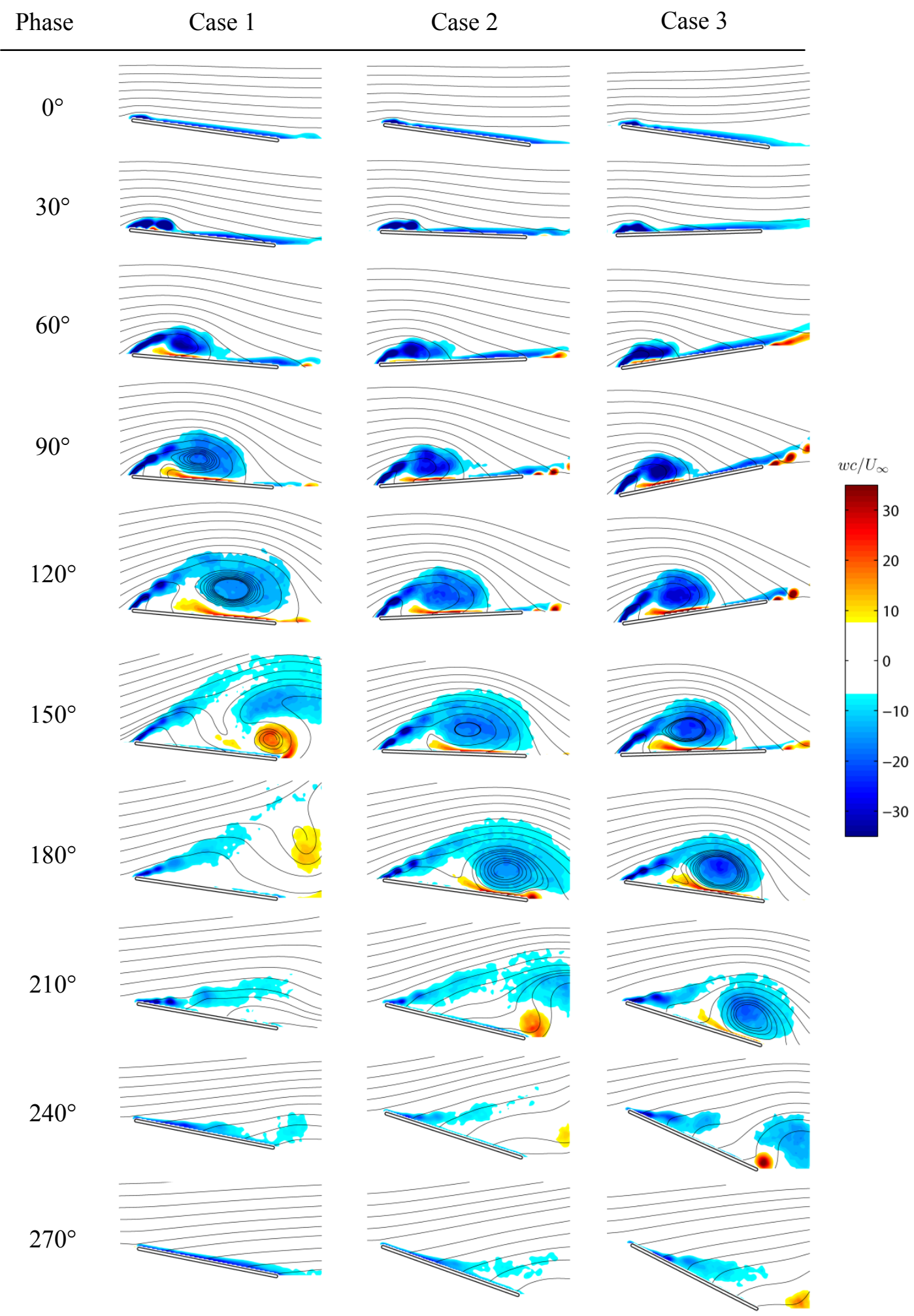

Figure B1 


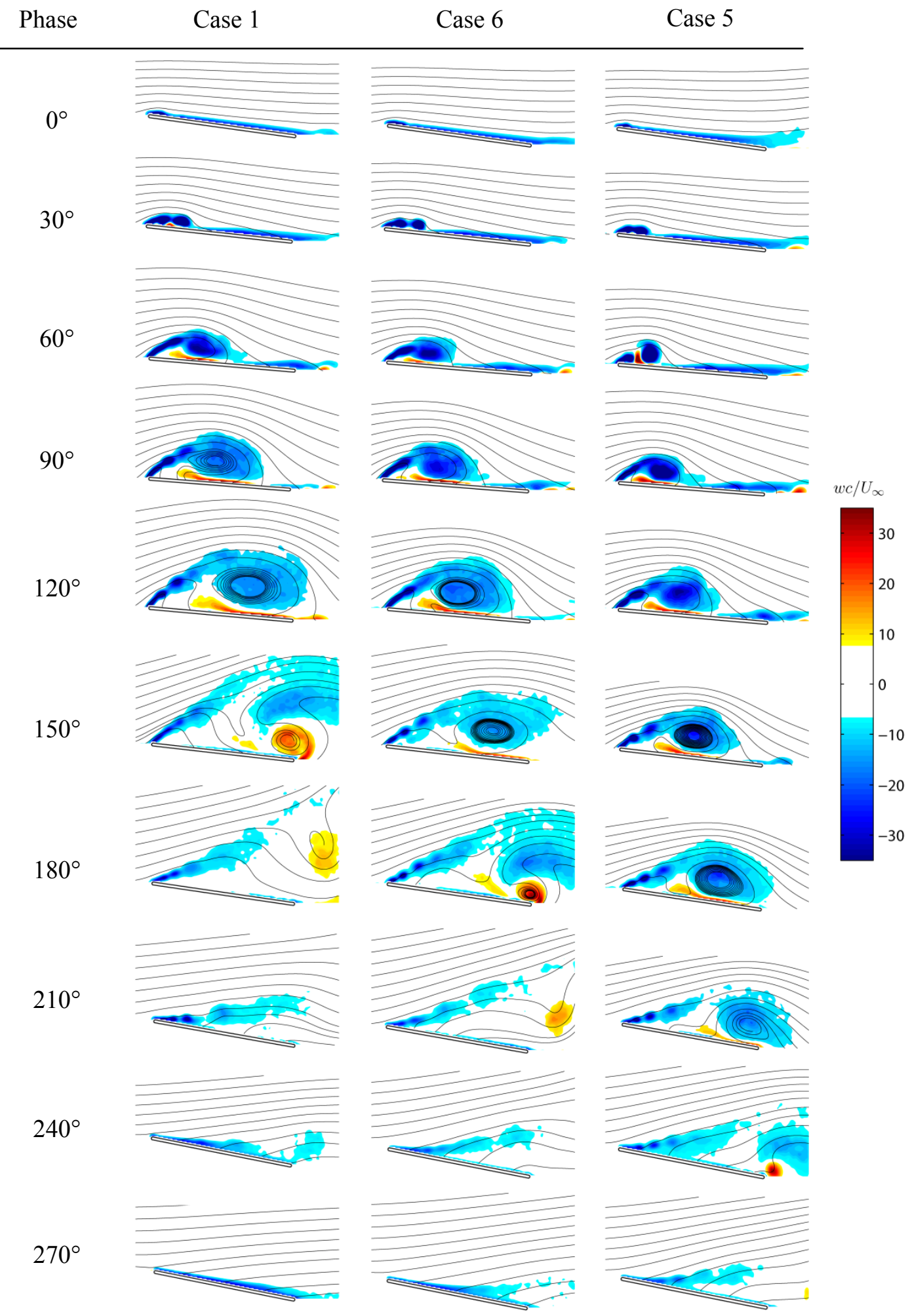

Figure B2 


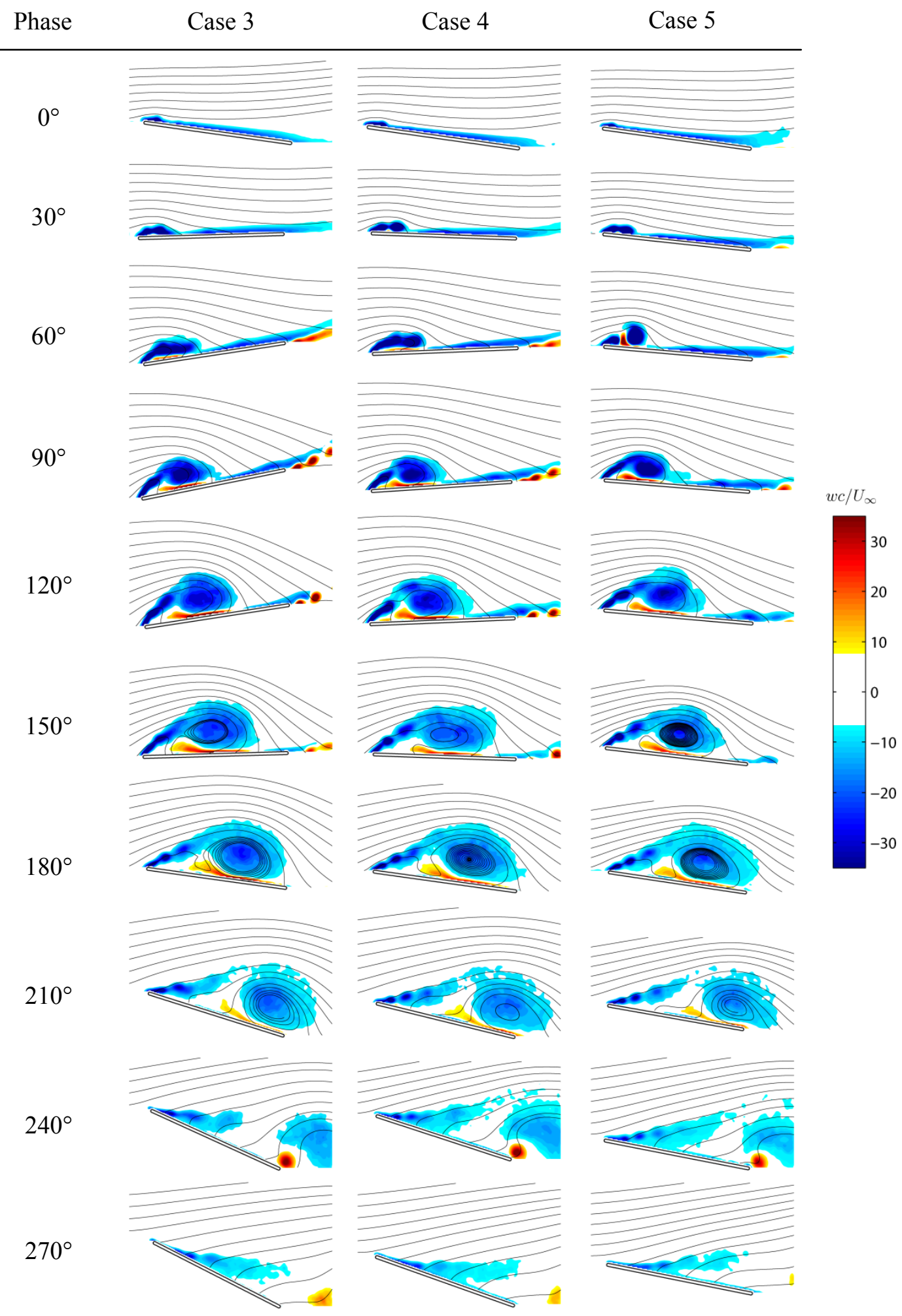

Figure B3 


\section{References}

1. Shyy, W., Lian, Y., Tang, J., Viieru, D., \& Liu, H. (2008). Aerodynamics of Low Reynolds Number Flyers. Cambridge University Press.

2. Platzer, M., Jones, K., Young, J., \& Lai, J. (2008). Flapping Wing Aerodynamics: Progress and Challenges. AIAA J. , Vol. 46, No. 9, 2136-2149.

3. Poelma, C., \& Dickinson, M. (2006). Time-Resolved Reconstruction of the Full Velocity Field around a Dynmamically-Scaled Flapping Wing. Experiments in Fluids, Vol. 41, pp. 213-225.

4. Sane, P., \& Dickinson, M. (2001). The Control of Flight Force by a Flapping Wing: Lift and Drag Production. $J$. Exp. Biol, Vol 204, pp. 2607-2626.

5. Usherwood, J., \& Ellington, C. (2002). The Aerodynamics of Revolving Wings I. Model Hawkmoth Wings. $J$. Exp. Biol, Vol. 205, pp. 1547-1564.

6. McCroskey, W. J. (1981). The phenomenon of dynamic stall. NASA TM-81264 .

7. Dickinson, M. H., \& Gotz, K. G. (1993). Unsteady aerodynamic performance on model wings at low Reynolds numbers. J. Exp. Biol , 45-64.

8. Ellington, C. P. (1984). The aerodynamics of hovering insect flight. IV. Aerodynamic mechanisms. Phil. Trans. R. Soc. Lond. B , 79-113.

9. Maxworthy, T. (1979). Experiments on the Weis-Fogh mechanism of lift eneration by insects in hovering flight Part 1. Dynamics of the 'fling'. J. Fluid Mech. , 47-63.

10. Freymuth, P. (1988). Propulsive Vortical Signatures of Plunging and Pitching Airfoils. AIAA J. , 881-883.

11. Koochesfahani, M. (1989). Vortical Patterns in the Wake of an Oscillating Airfoil. AIAA J., 1200-1205.

12. Jones, K. D., Dohring, C. M., \& Platzer, M. F. (1998). Experimental and Computational Investigation of the Knoller-Betz Effect. AIAA J. , 1240-1246.

13. Lai, J. C., \& Platzer, M. F. (1999). Jet Characteristics of a Plunging Airfoil. AIAA J., 1529-1537.

14. Ohmi, K., Coutanceau, M., Loc, T. P., \& Dulieu, A. (1990). Vortex formation around an oscillating and translating airfoil at large incidences. J. Fluid Mech. , 37-60.

15. Anderson, J. M., Streitlien, K., Barrett, D. S., \& Triantafyllou, M. S. (1998). Oscillating foils of high propulsive efficiency. J. Fluid Mech. , 41-72.

16. Taylor, G. K., Nudds, R. L., \& Thomas, A. L. (2003). Flying and swimming animals cruise at a Strouhal number tuned for high power efficiency. Nature, 707-711.

17. Ol, M. V., Bernal, L. P., Kang, C., \& Shyy, W. (2009). Shallow and deep dynamic stall for flapping low Reynolds number airfoils. Experiments in Fluids, 883-901.

18. Baik, Y., Rausch, J., Bernal, L. P., \& Ol, M. (2009). Experimental Investigation of Pitching and Plunging Airfoils at Reynolds Number between $1 \times 10^{\wedge} 4$ and $6 \times 10^{\wedge} 4$. 39th AIAA Fluid Dynamics Conference. San Antonio, TX.

19. Kang, C., Baik, Y., Bernal, L. P., Ol, M., \& Shyy, W. (2009). Fluid Dynamics of Pitching and Plunging Airfoils of Reynolds Number between $1 \times 10^{\wedge} 4$ and $6 \times 10^{\wedge} 4.47$ th AIAA Aerospace Sciences Meeting including The New Horizons Forum and Aerospace Exposition. Orlando, FL. 
20. Visbal, M. R., \& Shang, J. S. (1989). Investigation of the Flow Structure Around a Rapidly Pitching Airfoil. AIAA J., Vol. 27, No.8, 1044-1050.

21. Theodorsen, T. (1935). General Theory of Aerodynamic Instability and the Mechanism of Flutter. NACA Report 496

22. Ho, C., \& Huerre, P. (1984). Perturbed Free Shear Layers. Ann. Rev. Fluid Mech., Vol 16, pp. 365-424.

23. Jeong, J., \& Hussain, F. (1995). On the identificaiton of a vortex. J. Fluid Mech., Vol 285, pp. 69-94. 